\title{
Arquitecturas tradicionales y populares: un reto para la historiografía de la arquitectura en Colombia
}

\author{
Traditional and popular architectures: a challenge for architecture \\ historiography in Colombia
}

\section{Guillermo Gutiérrez-Morales}

Universidad Católica de Pereira (Colombia)

Facultad de Arquitectura y Diseño

\author{
Arquitecto, Universidad Nacional de Colombia, Manizales \\ (Colombia). \\ Antropólogo, Arqueólogo y Mg. Historia, Universidad del Cauca, \\ Popayán (Colombia). \\ Especialista en Educación, Universidad Católica de Manizales, \\ Manizales (Colombia) \\ Candidato a Doctor en Arte y Arquitectura, Universidad Nacio- \\ nal de Colombia, Bogotá (Colombia). \\ (iD) https://orcid.org/0000-000I-7889-52IX. \\ https://scholar.google.com/citations?hl=es\&user=Y- \\ 2jSv-T179sC \\ (a) guillermol.gutierrez@ucp.edu.co
}

Gutiérrez-Morales, G. (2019). Arquitecturas tradicionales y populares: un reto para la historiografía de la arquitectura en Colombia. Revista de Arquitectura (Bogotá), 22(2), 60-68. https://doi.org//0.14718/RevArq.2020.2040

\section{(c) (1) (9)}

\section{Resumen}

La mirada sobre las arquitecturas tradicionales - en este caso, de los pueblos indígenas misak y nasa, así como de comunidades negras, en el departamento del Caucatiene su razón de ser en la necesidad de reconocer la arquitectura propia de estos grupos humanos, para que hagan parte de la historiografía de la arquitectura colombiana. Se partió de investigaciones cuyo objetivo fue la reconstrucción crítica de las transformaciones de dichas arquitecturas durante el periodo de modernización del Estado-nación. Se hizo un ejercicio metodológico que integra la etnografía, la revisión de archivo notarial y prensa, de cartografía y planimetrías, levantamientos arquitectónicos in situ y cronotipologías de estas arquitecturas en el área urbana y rural de los municipios de Silvia y Suárez. Con esto se busca el reconocimiento de la casa como hábitat básico, donde está el punto de partida de la reproducción social con sus representaciones y simbolismos, como elemento estructural de la cultura de los grupos humanos asentados en la ruralidad del Cauca.

Palabras clave: Conocimientos tradicionales; cultura tradicional; investigación histórica; cultura; cultura indígena; patrimonio cultural; arquitectura vernácula;

\section{Abstract}

This reflection raises the need to redirect the look on traditional architectures, in this case of the Misak and Nasa indigenous peoples, as well as black communities in the Cauca department, a reflection motivated by the need to recognize the architecture of these human groups to be part of the historiography of Colombian architecture. The above based on two investigations whose objective was the critical reconstruction of the transformations of these architectures during the period of modernization of the nation-state. Methodological exercise that integrates ethnography, the review of notarial and press archives, cartography and planimetries, on-site architectural surveys and chronotypologies of these architectures in the urban and rural areas of the municipalities of Silvia and Suárez. What this seeks is acknowledging the house as a basic habitat, where the starting point of social reproduction lies with its representations and symbolisms, as a structural element of the culture of human groups settled in the rurality of Cauca.

Key words: Traditional knowledge; traditional cultures historical research; culture; indigenous culture; Cultura heritage; vernacular architecture;

\section{Introducción}

El presente artículo es uno de los resultados de dos investigaciones ${ }^{1}$ que se desarrollaron en el departamento del Cauca, en Colombia, dentro del marco de las tesis en antropología y de la Maestría en Historia, a partir de una inquietud inicial por conocer la arquitectura doméstica tradicional (figura 1) de este territorio multiétnico y pluricultural del país. Un territorio que, históricamente, ha acogido múltiples acontecimientos y coyunturas en la construcción de la nación, como los relacionados con la tenencia de la tierra. Tal situación en el territorio caucano ha dado lugar a oscilantes ciclos económicos y a crisis sociales localizadas de duración intermedia, en las que se han incubado procesos de resistencia soterrada de lo cultural, desde su base étnica, tanto en lo material como en lo inmaterial.

El tema de investigación sobre la arquitectura doméstica tradicional se trabajó con comunidades urbanas periféricas y rurales, con el caso del municipio de Silvia (Cauca), donde se encuentran pueblos indígenas paez y guambiano ${ }^{2}$, así como campesinos y habitantes urbanos mestizos, en un territorio referenciado administrativamente, pero no compartido. De igual manera, con la comunidad negra del Consejo Comunitario de Mindalá, en Suárez, donde la construcción de la represa de La Salvajina afectó, desde mediados del siglo XX, el hábitat de varios corregimientos del municipio (figura 2).

Se plantea, entonces, como objeto de estudio la arquitectura de la casa como producto de la

1 Proyecto 1: la arquitectura habitacional en Silva, Cauca (1930-1950).

Proyecto 2: la arquitectura doméstica de la comunidad negra del consejo comunitario de Mindalá, en Suárez, Cauca: poblamiento, hábitats e identidad.

2 Nasa y misak desde un reconocimiento emic. Los términos emic y etic fueron introducidos por el lingüista antropológico Kenneth Pike (1967) y han sido adoptados por los antropólogos sociales. En este caso, emic se refiere a cómo explican los miembros de la misma sociedad un fenómeno, una costumbre o un concepto propio. 
cultura, la casa tradicional en la ruralidad, que es repensada y reeditada desde lo histórico dentro del marco de lo étnico. Se plantea la necesidad de una mirada de "lo histórico-cultural", en torno a la casa tradicional rural, entendida como el constructo social-familiar que se configura a partir de la interacción de las personas en un territorio reconocido por ellas mismas desde la tradición oral y su experiencia de vida, donde se han construido históricamente respuestas a las condiciones particulares de existencia y donde, a partir de rituales y prácticas, se definen formas de vida constituidas desde la realización de actividades sociales y familiares compartidas.

Con estas premisas se define el tema de la presente investigación, teniendo en cuenta la arquitectura de la casa que surge a partir de la cultura, la historia social en el lugar, las condiciones particulares de habitar, y, por ende, de los hábitats de los pueblos indígenas nasa (figura 3) y misak en Silvia, así como de las comunidades rurales afrodescendientes del corregimiento de Mindalá, en Suarez, en el Cauca (figura 4). Con lo anterior se establecería que la casa en estos grupos no es neutral, pues en ella se incuban procesos de resistencia sociopolítica y cultural, así como de supervivencia y convivencia, entendiendo que el objeto de estudio es la casa, pero no solo en su noción material y funcional, sino aquella casa que puede ser descrita como receptáculo de prácticas sociales y culturales.

La motivación de la presente reflexión se establece a partir de esas posibilidades que reposicionan a quien investigue sobre arquitectura frente a la historia, debido a la posibilidad de interpretar las estructuras profundas presentes en el contexto histórico de la experiencia propia y ajena.

La relevancia de una relectura de la casa desde lo histórico-cultural remite a las múltiples interpretaciones posibles de ser y estar en un lugar. Allí confluye lo humano en intimidad y se gestan las concepciones idealizadas del ser. En la casa, con su espacialidad y su materialidad como escenario, las aspiraciones encuentran principios explicativos que le permiten al ser humano ordenar su mundo y configurar interpretaciones de lo que sucede por fuera de ella; esto es, la tragedia de la existencia.

Una panorámica general del problema que motiva esta reflexión, para la arquitectura, surge por los pocos trabajos historiográficos de la arquitectura tradicional elaborados con perspectivas regionales, de tipo localizado, rural o étnico; quizás, por la imposibilidad de manejar el territorio social colombiano, debido a la pluralidad entre regiones y en el interior de ellas mismas, como una unidad historiable (Arango, 2009, p. 34). Esto evidencia que no es posible construir una visión homogénea de las comunidades en los contextos regionales del país, ni, por tanto, de sus arquitecturas.

Igualmente, para la ruralidad y la periferia, como lo plantea para la ciudad Gutiérrez-Aristizábal (2017), es importante el reconocimiento de "la relación del hombre con su entorno físico y
(1) Figura 1. Casa tradicional misak en el resguardo de Guambia, Silvia (Cauca). Fuente: elaboración propia (2017). CC BY-NC-ND
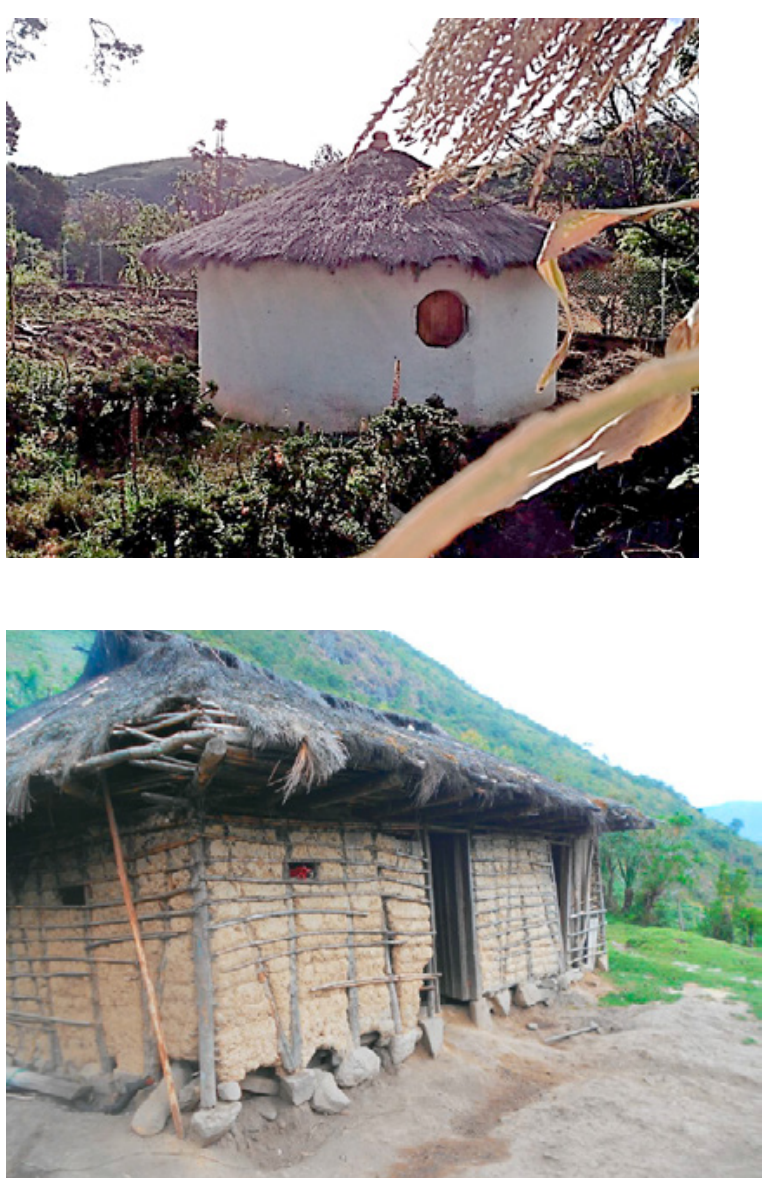

A Figura 2. Colombia.

Departamento del Cauca. Municipios de Popayán, Silvia y Suárez. Localización general. Fuente: elaboración propia (2017). CC BY-NC-ND

(A) Figura 3. Casa tradicional nasa en carrizo y bahareque. Páez (Cauca).

Fuente: elaboración propia (2017). CC BY-NC-ND

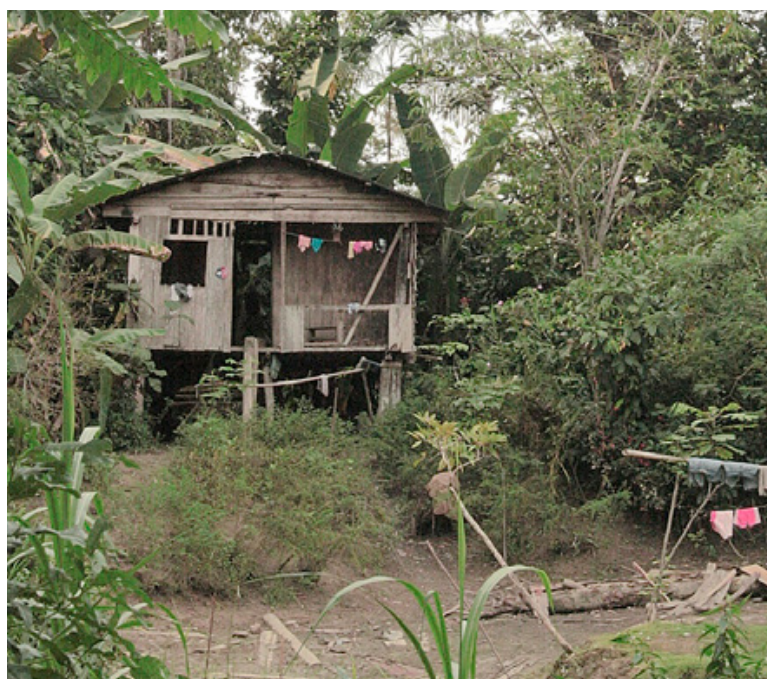

(A) Figura 4. Casa palafítica afrodescendiente. Suárez (Cauca). Fuente: elaboración propia (2017). CC BY-NC-ND social expresado en la construcción de su hábitat, [...] como referente uno de sus productos culturales más significativos" (p. 17), lo que se constituye en un tema estructural de investigación interdisciplinar para su reflexión y su discusión.

Es en su territorio en el lugar donde, más allá de la materialidad, emerge el hecho de habitar como condición que permite llegar a la expresión pura y abstracta del espíritu que la arquitectura acoge. Por lo tanto, se hacen necesarias reflexiones sobre el valor histórico-cultural de dichas arquitecturas, hacia una historiografía que las reconozca, que ayude a superar la marginalización de expresiones materiales construidas a partir de estructuras inmateriales vitales, como las presentes en la arquitectura de la casa rural tradicional. 


\section{Metodología}

En esta investigación, de carácter teórico y empírico, para establecer los principales referencias investigativas sobre la casa rural tradicional, se hizo una segmentación de la pregunta de investigación a partir de temáticas que, teórica y metodológicamente, permiten establecer lo hecho respecto a temas históricos, culturales, sociales y políticos que, de manera directa o alterna, se vinculan con la casa rural tradicional y su arquitectura de los pueblos indígenas nasa y misak, en Silvia, y las comunidades afrodescendientes del corregimiento de Mindalá, en Suárez, tanto en la particularidad del área de estudio como, de manera complementaria, en otras regiones que históricamente habitan estos grupos.

Para la comprensión de la arquitectura de la casa de los mencionados grupos humanos en el Cauca se retoma una estrategia metodológica a partir de la cual las ciencias humanas y sociales han abordado los análisis de procesos sociales. Por lo tanto, retomando a Reinhart Koselleck (2012), se establece que "la importancia de la experiencia es fundamental para situar la importancia del lenguaje, su interpretación y los significados que se derivan con el concurso de los conceptos" ( $p$. 29). Por consiguiente, se recurrió a estrategias y postulados de la historia cultural, la microhistoria, las historias de vida y la etnografía, así como a cronotipologías de ámbito local; estas últimas, desarrolladas especialmente para ambos momentos.

Como estrategia para el análisis de la realidad o las realidades posibles, en torno a la casa rural tradicional, se estableció la necesidad de generar un contexto histórico-cultural de los pueblos indígenas y las comunidades rurales afrodescendientes mencionados, ya que con ello se definieron los marcos teóricos, de referencia y de conceptualización de esa arquitectura de la casa rural tradicional. Finalmente se hizo una categorización de la revisión documental y de archivo, de lo recuperado a partir de la oralidad y los levantamientos de dicha arquitectura en sus diferentes contextos, para determinar, de manera panorámica, cuál sería el aporte de dichos referentes a esta reflexión.

Para lo correspondiente a lo empírico se hizo una reconstrucción de los momentos de la arquitectura habitacional tradicional de los pueblos indígenas nasa y misak y de las comunidades negras en las áreas objeto de estudio, teniendo presente lo sucedido hasta el establecimiento de la Constitución de 1991 y lo que se dio después de ella, punto de partida para la recopilación de información y el posterior análisis de las temáticas estructurales de la investigación: la sociedad rural indígena y afrodescendiente en el Cauca, lo histórico-cultural como elemento diferenciador y de identidad para la arquitectura de la casa.

Lo anterior se hizo por medio de una revisión bibliográfica y documental de los aspectos his- tóricos, socioculturales y legislativos. Posteriormente se desarrolló el trabajo de campo para la realización de levantamientos arquitectónicos de los asentamientos, para definir con esto los posibles procesos de poblamiento. Lo anterior se llevó a cabo con las referencias que se lograron establecer con las personas, desde la oralidad, en el territorio de los casos de estudio.

La localización de los pueblos indígenas y las comunidades rurales afrodescendientes en el Cauca permitió establecer variables diferenciales en lo histórico (poblamiento), lo arquitectónico (la casa) y lo sociocultural (prácticas) que permiten la definición de propiedades y atributos posibles de ser comparados. Comparación que es posible por lo étnico-cultural, pues ello establece un criterio base de homogeneidad. Variables diferenciales que, igualmente, dan línea para el establecimiento de los referentes teóricos y las precisiones necesarias de lo metodológico. Así mismo, fue necesario, para la formulación del problema, definir el contexto de justificación y el control de hipótesis, así la implementación del método comparativo, a partir de la recopilación, la sistematización y el análisis de fuentes primarias y secundarias.

La apuesta metodológica de estos ejercicios se constituye, inicialmente, por una necesidad dialógica interdisciplinar entre la arquitectura y las ciencias humanas y sociales, situación que requirió una reflexión acerca de los instrumentos y los métodos particulares con los que la historia, la antropología y la arqueología estudian la arquitectura; esto, debido a que la arquitectura tradicional, dada de manera informal, rural y urbana periférica, no ha estado en la lista de intereses de los "historiadores de la arquitectura": en la mayoría de los casos, la arquitectura rural tradicional se ha abordado desde la antropología y la arqueología, no como eje de investigación, sino referenciando a esta como producto de la cultura.

Por último, los casos de estudio se establecieron con una perspectiva metodológica transdisciplinar (arquitectura, antropología, historia) donde se entiende que el conocimiento es el resultado de un proceso de construcción o reconstrucción de la realidad que tiene su origen en la interacción entre las personas y el mundo (Bautista, 2011, p. 47). Esto permite la implementación del método comparativo (como ya se mencionó) como estrategia de los estudios cualitativos, estrategia propia de las ciencias humanas y sociales donde se integran la argumentación teórica y las evidencias empíricas.

En síntesis, se buscó reconocer la arquitectura de la casa rural tradicional de pueblos indígenas y comunidades negras en parte de la ruralidad del departamento del Cauca a partir de un correlato disciplinar. Esto, debido a que, actualmente, en dichos territorios hay presencia de sincretismos arquitectónicos y préstamos culturales que han dado origen a nuevas concepciones y construcciones de hábitat. Allí se reconfigura el hecho 
de habitar desde nuevas identidades fronterizas y mestizajes arquitectónicos en la periferia; además, se evidencia la aparición de un nuevo locus social culturalmente aprehendido.

\section{Resultados}

La perspectiva con la que se abordó el problema de la historiografía de la arquitectura tradicional, constituida a partir de las categorías de Estado-sociedad-cultura, establece un acercamiento al pasado cercano de la casa tradicional como territorio y espacio de construcción del ser. Es allí donde hábitat y habitar se configuran, inicialmente, desde lo cultural y lo social.

Cabe señalar, con lo anterior, que los cambios estructurales en la sociedad son propiciados de manera lenta por una serie de movimientos - unos, perceptibles; otros, no tanto- por parte de las élites detrás de la figura del Estado, como es el caso de tenencia de tierras y el cambio de los modelos de explotación por fuera de una economía de recursos, y sin ser esto una interpretación marxista.

Dentro del contexto descrito es posible identificar que las estrategias de desarrollo, desde los imaginarios de modernización en la primera mitad del siglo XX, generaron cambios de fondo $y$ forma que indujeron a las comunidades rurales $y$ urbanas periféricas a una condición de marginalidad promovida por el propio Estado. Y podría decirse que, si bien el Estado se encuentra en la misma sociedad, su praxis no ha sido equitativa ni este es racionalmente pensado por todos.

Durante los últimos treinta años se han fortalecido los ejercicios investigativos al vincular a la arquitectura, con mayor fuerza, aspectos sociales y culturales. En estos se reconstruyen relaciones de poder a partir de los recientes estudios culturales planteados dentro de algunas ciencias sociales. Se han enriquecido los documentos mediante el uso de imágenes y elementos gráficos de los que hace uso la arquitectura, y con los que se ha podido ir más allá de las clasificaciones estilísticas y se ha logrado llegar a profundizar en los análisis tipológicos y topofílicos. Se hacen presentes, además, trabajos interdisciplinarios donde la condición intangible de habitar remite a nuevas comprensiones del sentido de este y de la arquitectura.

Además de lo anterior, vale la pena precisar que la arquitectura popular, reconocida historiográficamente, solo aparece en escena en el momento en que se consolida la "alianza arquitecto-Estado durante los años 60 del siglo XX" (Solo, 1987, p. 30), tras el inicio de la urbanización del territorio y la configuración de la vivienda como un problema de mercado; ello deja claro que la casa, como arquitectura tradicional dada de manera informal, rural y urbana periférica, no estaba en la lista de intereses de los historiadores de la arquitectura; por el contrario, se abordó más el tema desde la antropología, no como eje de investigación, sino como producto de la cultura.

Es importante, entonces, buscar, para la historiografía de la arquitectura, en los análisis tipológicos y topofílicos la complementariedad y el diálogo interdisciplinar, para así reconocer el hecho de habitar y sus diferentes manifestaciones. De ahí que sea necesario establecer que habitar va más allá del sentido de resguardarse o alojarse, y esto es, en primer orden, reconocer las condiciones cualitativas espirituales desarrolladas de manera alterna a las cuantitativas materiales. Con lo anterior es posible establecer desde lo cultural las variadas realidades que dotan a al hecho de habitar de múltiples representaciones y significados.

\section{Discusión}

En esa ruta establecida para el conocimiento y la comprensión de las arquitecturas tradicionales en el departamento del Cauca, se devela un primer prejuicio disciplinar respecto a estas. Dichas arquitecturas, por ser sojuzgadas, de cierta manera, en un nivel de "minoría de edad" ${ }^{3} \mathrm{o}$ de estar en el orden material de lo perecedero, son consideradas inferiores y con menos valor que las denominadas arquitecturas históricas. Un concepto, además, que sería replicable en el nivel o en el reconocimiento de la producción material de construcciones para la habitación realizadas por parte de los subalternos sociales, étnicos y culturales; esto, desde las visiones academicistas eurocéntricas, que, en su momento, no las reconocían como arquitectura, pues para ser definidas como tal, quienes las producían debían estar en camino, por decirlo de alguna manera, de "modernizarse".

Uno de los prejuicios ante estas "arquitecturas sin arquitectos" se da al estar por fuera de las configuraciones ontológicas surgidas del proyecto colonizador capitalista que, desde metáforas del ser y el estar, ha instituido parámetros inquisidores de valoración y reconocimiento. Conviene advertir que con ello se establecen las supuestas verdades posibles; y es dentro del marco de dichas verdades posibles como la historia de la arquitectura se ha escrito.

Se ha presentado, podría decirse, un sesgo analítico, pues mucha de la historiografía de la arquitectura ha acogido la violencia simbólica propia del orden imperante al no reconocer el "descentramiento antropocéntrico de la forma humana de ser-ahí-en-el-mundo, propia de otros sistemas culturales interactuantes en la composición multiétnica y pluricultural" (Rodrigo Arango, en Barona y Rojas, 2007, p. 14) de los territorios que conforman, para este caso, la nación colombiana.

3 El concepto de "minoría de edad" para el siglo XIX es utilizado en la Ley 89 de 1890 referido a los "indígenas", lo que los presenta como en camino a la vida civilizada y los subordina a un "control paternal". 
Por lo planteado, una postura necesaria de reconocimiento de la tradición arquitectónica, como saber colectivo, remite tanto a lo personal y a lo común — por las particularidades propias del individuo y el lugar - como al diálogo con las generalidades de sujeto y contexto. Lo anterior debe ser reconocido desde la historia y la historiografía en temporalidades asimétricamente complementarias, lo local y lo regional, el pasado lejano y el pasado reciente. La historia de la gente en sus espacios vitales cotidianos.

Es necesario, entonces, establecer los elementos tangibles e intangibles presentes en la arquitectura tradicional que, como lo expone el antropólogo Marvin Harris (1982), con su perspectiva del materialismo cultural, se pueden abordar desde una construcción teórica fundada en principios epistemológicos, lo que, a su vez, daría a estos elementos el reconocimiento en su papel de dar sentido a dicha arquitectura como construcción de territorio de esos otros desde el pasado. Lo anterior, con el fin interpretar en el marco de la diferencia y la base empírica que los acompaña, los valores propios y elementos de significación, para su reinterpretación y su reedición, valores que se han desvirtuado debido al desencuentro cultural existente.

Analizar los aspectos que confluyen, por ejemplo, en la casa o en la vivienda, como materialidad y representación, con la perspectiva materialista cultural, se deja abierta la posibilidad del uso de estrategias alternativas de reflexión y comprensión de los fenómenos sociales y de lo cultural que Harris (1982) denomina "otros modos de conocimiento" manifestando "[...] que no tiene inconveniente en admitir que existen ámbitos de la experiencia a los que no se puede acceder por medio de la adhesión a las reglas del método científico" (p. 21), por lo cual el análisis de la concepción de la casa y su representación a partir de su uso, su distribución y su zonificación, así como su materialización, permite el conocimiento de aspectos del medio y de las dinámicas sociales que han influido en el devenir de la comunidad y la construcción de lo cultural desde la experiencia del pasado.

Además de lo anterior, el estudio de la arquitectura tradicional, desde lo cultura, material e inmaterial, permite hacer una lectura del desarrollo del poder económico y su reproducción; ambos, sobre bases ideológicas. Con ello se establece la capacidad de los grupos hegemónicos (Estado y élites) para hacer uso de lo simbólico a fin de perpetuar sus ideologías y mantener el control. Es aquí donde se puede evidenciar la mayor eficacia de lo simbólico por encima del propio poder económico. Las arquitecturas tradicionales y populares se constituyen en los elementos del hábitat que de manera material evidencian el papel de lo simbólico y su origen.

Entonces, la relación entre los pueblos inmersos en procesos de construcción de memo- ria colectiva y el territorio se "establece con la manifestación de actividades vinculadas a espacios físicos y temporales representativos, que se reproducen de manera eventual en las prácticas sociales; allí es donde se establecen los valores del pasado y la necesidad de mantenerlos en el tiempo" (Gutiérrez, 2012, p. 993). En este sentido, por ejemplo, en el territorio del departamento del Cauca, para el pueblo indígena nasa ${ }^{4}$ un mismo sintagma recoge varias ideas o entidades que, a su vez, están particularizadas por el ámbito de realidad de estos. El lingüista Tulio Rojas (2007) plantea que

\begin{abstract}
(...) nasa remite al conjunto en el cual el individuo se encuentra inmerso, que lo identifica como uno de sus miembros y a la vez él mismo co-actúa con el grupo y co-define su identidad. Conjunto que en primer acercamiento es con los miembros de la comunidad con la cual se convive en forma permanente, en una segunda aproximación a todos aquellos que forman parte del mismo pueblo y en una tercera esfera de influencia es el conjunto de los grupos humanos. (Barona y Rojas, 2007, p. 125)
\end{abstract}

Además de lo anterior, "[...] el término nasa, pero con el sufijo -sa indica una reafirmación del concepto, el cual da un sentido particular de identidad — propiamente nasa- frente al sentido genérico de nasa de la primera"; esto es, nasasa. Se establece entonces que...

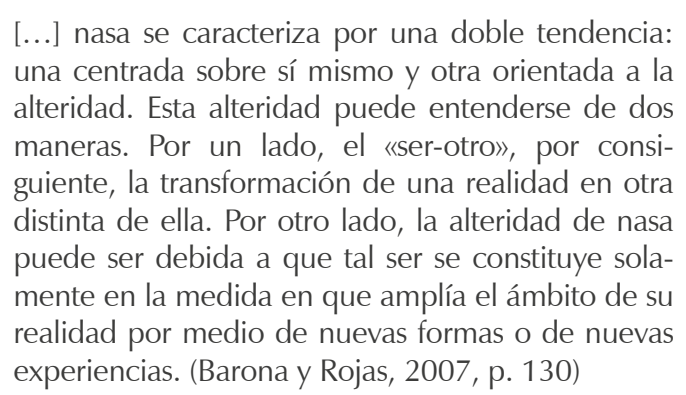

Por lo tanto,

[...] el término nasa, que en primera instancia se asimilaría a «gente», no denota sólo la humanidad como especie natural ni tampoco exclusivamente su condición social y su ámbito referencial involucra además de los humanos a otros seres del mundo. El concepto de nasa está absorbido en todas las entidades y al mismo tiempo por encima de ellas trascendiéndolas. (Barona y Rojas, 2007, p. 129)

Con lo anterior como ejemplo desde la lengua, en conexión con la construcción de esas realidades de mundo o de origen, y que constituyen la representación de las cosas evidenciadas en la materialidad, se puede inferir que la transformación del concepto de casa, en el contexto de la arquitectura tradicional, como territorio ha permitido resignificar, debido a una nueva complejidad, el término lugar. Ahí subyacen complejas estructuras intangibles del espíritu individual y colectivo, concepto desarrollado en una diacronía Ilena de situaciones que debilitan, fortalecen

4 Conocidos como paeces, el pueblo indígena nasa, desde una posición emic, se consideran y se reconocen a sí mismos como originarios del territorio que constituye el suroccidente colombiano, y lo habitan, al igual que otros pueblos indígenas, en resguardos legalmente constituidos. 
o transforman el espacio básico de habitación, y que Bourdieu (2007) recoge en lo que denomina

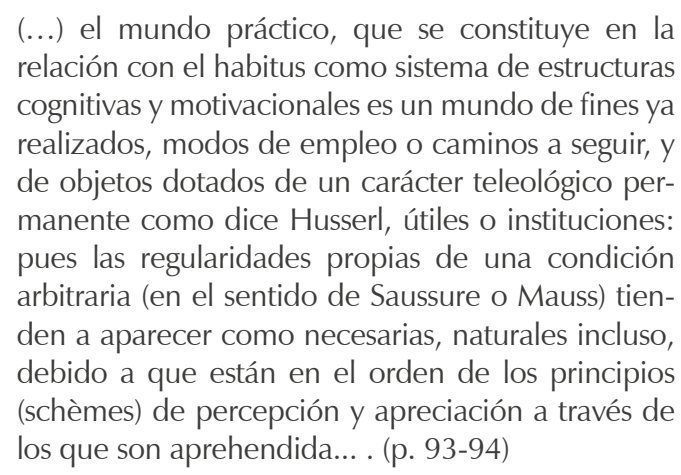

(...) el mundo práctico, que se constituye en la relación con el habitus como sistema de estructuras cognitivas y motivacionales es un mundo de fines ya realizados, modos de empleo o caminos a seguir, y de objetos dotados de un carácter teleológico permanente como dice Husserl, útiles o instituciones: pues las regularidades propias de una condición arbitraria (en el sentido de Saussure o Mauss) tienden a aparecer como necesarias, naturales incluso, debido a que están en el orden de los principios (schèmes) de percepción y apreciación a través de los que son aprehendida... . (p. 93-94)

Realidad manifiesta en la memoria que redefine constantemente el espacio-tiempo y da la mano a la experiencia expresada en el texto o la tradición oral que, a su vez, es constituida dentro de los espacios de la cotidianidad; es decir la casa.

Así pues, en ese paso del habla al texto, con la noción de causalidad que le antecede, se establece que, semánticamente, las palabras conducen a significados y a representaciones mentales. Dichas representaciones se animan en el espacio y la materialidad de los lugares de la vivencia, lugares que invitan a la construcción de pensamiento y a la comprensión del universo del ser, por lo que, en palabras de Bourdieu (2014) "[...] no existe un agente social que no aspire, en la medida de sus medios, a ese poder de nombrar y de construir el mundo al nombrarlo" (p. 81); de ahí que, como lo plantea Rodolfo Arango en el prólogo de Falacias del Pluralismo Jurídico y Cultural en Colombia, de los profesores Guido Barona y Tulio Rojas (2007), "[...] es un desafío epistemológico el comprender al otro cultural en su diferencia a partir de la lengua como casa del ser, esto en sentido Heideggeriano" (p. 15).

Los lugares de vivencia, entonces, se manifiestan en la ocupación del territorio y la presencia en este. Lo anterior, a partir de procesos sociales, económicos, políticos y de explotación que han generado o degenerado el moldeado físico y biológico del paisaje habitado y su imagen, su significación y su expresión. Esto, de una u otra manera, incide en el espacio básico de refugio, vida íntima y social de nuestra especie; de ahí que sea necesaria una aproximación a dicho espacio, pues allí es donde se concentra la vida cotidiana en sus variables material y simbólica, aspectos que De Certeau (1999) recoge cuando conceptualiza, de igual manera, el término territorio a una escala intima de cotidianidad, al considerar que:

[...] donde se despliegan y se repiten día con día las acciones elementales de las artes de hacer, es de entrada el espacio doméstico, esta vivienda a la que uno desea ardientemente retirarse, porque allí se conseguirá la paz. Uno regresa a su casa, a ese lugar propio que, por definición, no podría ser el lugar ajeno. Aquí cualquier visitante es un intruso, a menos que haya sido explícita y libremente convidado a entrar (p. 147).
La representación y el significado del hábitat básico - la casa tradicional donde la experiencia consiente e inconsciente del ser encuentra su base material-, como resultado de la construcción social e histórica compartida, está influida por la lengua en la cual la interacción de los individuos y la manipulación que hacen de esta permiten la pervivencia y el dinamismo de la base semántica que designa de alguna manera a los objetos, y donde la historia da herramientas para examinar dicha dimensión social, como ya se mencionó, entre olvidos y recuerdos.

El reflejo del ser y el estar de los grupos, familias e individuos en el diario actuar y pensar se ve reflejado de manera innegable en todas y cada una de las afectaciones materiales que han dejado huella en el territorio, recreación de mitos y referencia de significados que por medio del rito han trascendido en el tiempo, y que, a pesar de la aculturación por los procesos de colonización y evangelización o de los apadrinados por la globalización en la actualidad, se deben reivindicar para el mantenimiento de los valores y el autorreconocimiento. Esto último, desde la tradición oral y la recreación en el rito. En este sentido, por ejemplo,
[...] dependiendo de las condiciones del medio y de los mitos a través de los cuales se explicaron el mundo, cada grupo creó su vivienda: gigantescas malocas en las selvas amazónicas, pequeñísimos bohíos de base rectangular en las laderas de la sierra nevada del Cocuy, medianas construcciones circulares en los valles interandinos, empalizadas y casas en los árboles en las laderas del cauca medio, altos palafitos en las llanuras inundables del Atrato y el Magdalena [...] entre muchas más. (Duque, 2004, p. 24)

En la tradición oral o en el texto escrito el símbolo no es estático, y en el acto interpretativo el significado admite esta misma cualidad, pues las circunstancias del contexto permiten variaciones desde la conectividad diferencial dada en el tiempo y la experiencia, lo que facilita la interpretación de los significados a partir de procesos de negociación de estos. Y para la casa, como representación dispersa o agrupada en un territorio,

[...] el conjunto simbólico justificaría plenamente esta desconcertante perspectiva, mediante la aproximación de unidades simbólicas independientes las unas de las otras, y haría aparecer claramente la lógica que las reunía. Entonces esta búsqueda, en principio empírica, experimental y casi lúdica de les lieux de mémoire desembocaría en dos perspectivas infinitamente más excitantes: hacer de esta noción, ensamblada por necesidades de la causa, una categoría de inteligibilidad de la historia contemporánea, sino un concepto y cosa bastante rara en historia; contribuir a instituir una historia de tipo simbólico, que respondiera más que la historia clásica, a las necesidades científicas y cívicas de nuestro tiempo... . (Nora, 2008, p. 19)

La necesidad de un reconocimiento de los simbólico y lo cotidiano y la ruptura que se da en la labor historiográfica tradicional, fundada en la 
epistemología clásica, surge de una posición tomada por quienes se alejaron de los lineamientos de la estructura racional moderna que hasta finales del siglo XX regía la concepción de historia y su supuesto cognitivo, y formulada a partir de realidades occidentales de crecimiento y desarrollo progresivo, constante e indefinido de esta cultura, autodenominada civilizatoria. Se da un movimiento reaccionario que busca repensar el ejercicio disciplinar de la historia a partir de una nueva posición del hombre como sujeto de estudio desde la representación de su experiencia, a partir de relaciones temporales y de contexto.

Se hace necesario, entonces, reeditar el concepto de historia, que se presenta como una secuencia inquebrantable de procesos invariables ajenos al ser, independientes en sí mismos e inmutables dentro de una progresión cronológica establecida desde los imaginarios hegemónicos occidentales. Es aquí donde la noción epistémica de verdad, que para Foucault (1999) - vinculada esta al orden del lenguaje, y este, a su vez, en el orden de lo simbólico- actúa sobre la realidad determinándola, y soslaya las posibilidades dadas desde diferentes concepciones de mundo — de otros órdenes racionales- constituidos a partir de una base conceptual de cultura más amplia y multivocal. Reflexión pluralista que no significa llevar a una fragmentación el discurso o la comprensión de la verdad, en aras de una emancipación de realidades posibles dentro de la sociedad; si así fuera, esto facilitaría una fractura en el interior de ella, en tanto no reconozca su realidad pasada y la manera como se comprendió desde la tradición historiográfica clásica.

Por lo planteado, las condiciones que presentan las dinámicas actuales no deben sustraer a la historia de la arquitectura tradicional de la realidad presente ni, mucho menos, negarle la posibilidad de que sea útil en la visión y la construcción de futuro. Por el contrario, se hace necesaria una ampliación del espectro en el que la historia tiene un papel importante en la interpretación de la cultura; o quizá, mejor, retomando a Eduardo Restrepo (2012),

[...] cambiar el uso de la palabra cultura para hablar de lo cultural... es decir el desplazamiento de la formación sustantivada (cultura) a la adjetivación (lo cultural) permite enfatizar que los análisis se refieren más a una dimensión o característica de cualquier práctica o relación social que a una cosa en-elmundo como la palabra cultura sugiere... . (p. 33)

Cambio del sentido y el uso de la palabra cultura que se puede lograr desde la interacción disciplinar donde la historiografía pueda ser desarrollada por perspectivas opuestas, y quizá complementarias, a partir de la construcción del ya mencionado correlato empírico.

Debe considerarse, entonces, que con la complejidad de las dinámicas sociales, presentes en territorios como los del departamento del Cauca, son necesarios acercamientos generales y específicos a las prácticas en torno a la casa, debido a la heterogeneidad cultural, la diversidad étnica, las formas de inserción en el territorio y la pluralidad dentro del complejo y ambiguo "mundo de lo rural" ${ }^{5}$ y de lo urbano periférico, donde la condición ontológica material determina la construcción de conocimiento desde el habitus en cada territorialidad. Esto, según Bourdieu se refiere a los esquemas de obrar, pensar y sentir "asociados a la posición social, es decir, el habitus hace que personas de un entorno social homogéneo tiendan a compartir estilos de vida parecidos" (Bourdieu citado por Domínguez y Portellano, 2011, p. 153) en cada territorialidad, lo que permite, así, determinar lo específico y lo general de la sociedad campesina, "indígena" y "urbana periférica", para este caso.

Así pues, la articulación de lo anterior, desde las ciencias humanas y sociales, con la arquitectura, aunque para algunos suena como una especulación, no es una posibilidad inmovilizadora. Es posible una reconstrucción histórica con el uso de las arquitecturas populares y tradicionales, tal como lo plantea Koselleck en su libro La interdisciplinariedad de la historia (citado por Sánchez-Prieto, 2012), aunque con una perspectiva de temporalidad o periodicidad histórica, cuando

\footnotetext{
[...] arma dos categorías - espacio de experiencia y horizonte de expectativa- de enorme potencial explicativo (más allá de la historia de los conceptos o de un periodo histórico determinado) y que permiten al historiador distanciarse de cualquier historia teleológica, que reciba su sentido del exterior, en la manera de hacer la historia... . (p. 486)
}

Categorías que metodológicamente se pueden tomar como conceptos para la construcción de nuevas posibilidades de interpretación de verdades históricas alternas en una sinergia interdisciplinar, que se da no solo entre lenguaje y conocimiento, sino, además, a partir de la experiencia y de una posible visión no remota del sujeto y el objeto de estudio.

Cabe señalar, además, que las pautas de modernización han generado ritmos acelerados que desdibujan las posibles realidades de mundo, lo que permite una mayor y más fácil manipulación hegemónica. Se da la necesidad de tomar una posición analítica integral para la comprensión heurístico-hermenéutica de los procesos sociales (económicos, sociales, políticos y de lo cultural), debido a su magnitud, su distribución global y su capacidad de transformación de la arquitectura habitacional tradicional, el hábitat rural y el urbano periférico.

De ahí que los diferentes fenómenos causados por las variadas dinámicas que constituyen las realidades del Estado, la sociedad y la cultura en los territorios de un país como Colombia

5 Concepto utilizado de forma reiterada por la antropóloga Virginia Gutiérrez de Pineda (1958, p. 17) 
no deben analizarse con una sola perspectiva ni establecerse a partir de la formulación de generalizaciones; ello, debido a que los agentes, desde lo cultural y lo social, establecen procesos particulares como respuesta a las políticas y los proyectos del Estado según las condiciones y la oferta del contexto, sin ser esto último determinismo ambiental.

Lo anterior, para el caso de la casa, de la arquitectura tradicional como espacio doméstico o cotidiano, está construido de manera sobrepuesta, desde la marginalidad respecto a las políticas estatales por parte de las propias comunidades; ello, en razón de una temprana transferencia de la responsabilidad del Estado en lo tocante a la oferta (producción y financiamiento) de la denominada vivienda social, que fue puesta en manos de los propios individuos.

Se tiene, además, el papel de lo político y lo económico en torno a la cultura como condicionantes que influyen en la arquitectura tradicional de los procesos sociales y dan lugar a líneas de ruptura, o cleavage, según el politólogo noruego Stein Rokkan (Lipset y Rokkan, 1967). Allí, el de centro/periferia es

[... el eje fundamental en la línea de ruptura entre los esfuerzos de expansión y control del centro, evaluados en términos de recursos, distancias y canales de comunicación, y los esfuerzos de la periferia para resistir la dependencia y la pérdida de control de su destino. (Arnoletto, 2007, p. 53)

Según este último autor citado, dicha ruptura se da, si se prefiere, entre la cultura central para construir nación y la resistencia de las poblaciones periféricas, de cultura local no reconocida. Esta línea de ruptura centro/periferia, junto a la de Estado/Iglesia, ha tendido a producir desarrollos divergentes entre los diversos países o territorios.

Aunque Rokkan basa sus estudios en los países de Europa Occidental, planteados con una perspectiva histórica de larga duración, es posible vincular el concepto de centro/periferia que maneja como eje fundamental para el estudio de las condicionantes, ya mencionadas, a los procesos en Latinoamérica y Colombia, que, incluso en la actualidad, están dados por una dependencia administrativa centralista. Aun así, lo que parece un desarrollo regional dependiente, el acogimiento condescendiente de políticas del Estado o la participación limitada de la sociedad civil se fractura o se desvirtúa por la construcción sociocultural de la vida cotidiana según las estructuras culturales —representación y simbolismo- de base que se materializan en el habitar y en las arquitecturas tradicional y popular.

Según lo planteado, el papel de la sociedad y la cultura dentro del sistema implementado por el Estado es determinado por las crisis vitales que en ellas se presentan. Desde allí, sociedades y culturas buscan recuperar el equilibrio que permite su supervivencia, situación que se evidencia por las conductas y las respuestas emergentes en las maneras de habitar, en la reedición de los modelos de apropiación del territorio y la reconfiguración material de la arquitectura tradicional y la popular, a partir de su representación y su simbolismo; lo anterior presupone una manera diferente de como las partes de un sistema, por fuera de concepciones estructuralistas, construyen para equilibrarse.

Lo que aquí se establece como cultura periférica y en la periferia, mediante una visión con la perspectiva del otro, permite comprender una noción extensa del concepto cultura en el tránsito de una sociedad hacia la modernización, y que, por el fenómeno urbanizador que caracteriza al siglo pasado, promueve la necesidad de tener en cuenta las consideraciones históricas necesarias por la condición de un territorio que acoge pueblos y comunidades con niveles de desarrollo desigual, donde la cotidianidad y las arquitecturas tradicionales y populares son los custodios de lo cultural y la supervivencia social.

\section{Conclusiones}

El problema de entender el pasado construido por una sociedad y concebido desde la cultura debe establecer la distinción entre historia y tradición referida a la arquitectura, pues, como lo exponen Fonseca y Saldarriaga (1992) "[...] la arquitectura histórica es el hecho que nunca se repitió, la arquitectura tradicional es la continuación en el presente de una forma de construir del pasado" (p. 23). Por lo tanto, ambas arquitecturas, como manifestaciones del pasado, deben ser estudiadas en un proceso dialéctico de oposición y complemento.

Para acercarse a ello debe establecerse, desde la historia, que habitar va más allá del sentido de resguardarse o alojarse, y esto es, como primera medida, reconocer las condiciones cualitativas espirituales desarrolladas de manera alterna a las cuantitativas materiales. Con lo anterior es posible establecer, desde lo cultural, las variadas realidades que han dotado a habitar de múltiples representaciones y significados en el tiempo.

Así mismo, para una nueva historiografía de la arquitectura tradicional son necesarias la reivindicación y la valoración de las fuentes primarias y de la historia oral como instrumentos que permiten las posibles escrituras de otras posibles historias. Ahora bien, para la historia oral, aunque el paso del habla a la escritura refiere cierto nivel de "separación [...] no quiere decir que sea absoluta, ya que la escritura todavía es gobernada por la dialéctica del acontecimiento y del sentido" (Duque, 2012, p. 70); de hecho, el discurso reposa en el registro escrito y en el no escrito de las posibles reconstrucciones históricas entre el recuerdo y el olvido.

Como resultado de lo anterior, la conciencia histórica no debe ser limitada por las concepciones hegemónicas lineales de tiempo (orden cronológico) y estilo de narración propios del 
Occidente eurocéntrico. Por ejemplo, la recreación del mito desde el relato al texto puede estar yuxtapuesto con nociones de causalidad que evidencian vínculos con el pasado. Así pues, quizá, con una nueva historiografía de la arquitectura se pueda recuperar la razón de ser de la arquitectura: las personas; $y$, en palabras de Norbert Elías, su "morar en la tierra" (Silva, 2012, p. 127).

Reconocer los mestizajes culturales y las identidades constituidas en procesos de coexistencia de grupos de personas que se distinguen por tener encuentros en la diferencia. Procesos en los que se ha dado una filiación histórica entre individuos y territorio, y donde los primeros han recurrido a una gestión de la alteridad, de orden cambiante y múltiple, para la supervivencia sociocultural.

Lo anterior, desde la lógica misma de la identidad, da lugar a que las realidades de vida remitan a los individuos en comunidad a una configuración del hábitat, desde la arquitectura tradicional, donde sus búsquedas promueven diálogos que permean las identidades esenciales inamovibles y superan los pasados reificados. Estas fisuras de la cultura que se dan en contextos de mestizaje de manera ambivalente, por estar en terrenos del multiculturalismo, dan lugar también a interpretaciones sesgadas que remiten a la alteridad a diferencias insalvables que, desde la interpretación histórica y su posterior escritura, excluyen a aquellos que no pueden producir o reproducir las "pruebas civilizatorias" de los imaginarios occidentalizados.

Por lo anterior, en esos mestizajes la cultura material de un grupo no es estática ni se da solo por la imposición de imaginarios, no es el resultado de la elaboración mecánica de objetos que cumplen una función específica; es decir, solo utilitarios. Dicha cultura material (arquitectura tradicional) representa universos simbólicos que, en el orden de lo estético y lo poético, se han configurado a partir del descubrimiento y el redescubrimiento de técnicas, de la interiorización y la comprensión de sus realidades de vida espaciotemporales y la experiencia transmitida desde la oralidad. Y la casa como producto de la cultura no es ajena a ello, pues, como lo expresa Amos Rapoport (1972), "...la casa era como un microcosmos [...] un imago mundi" (p. 72).

Las arquitecturas tradicionales y populares, como producto material de la cultura desde el pensamiento, recogen las actitudes simbólicas que explican el predominio de la distribución simbólica del espacio en la casa y de la ubicación de esta en el territorio. Dichas arquitecturas, relegadas hasta hace pocos años, se constituyen en mecanismos sociales y culturales de resistencia y supervivencia, pues, entre lo diferente y distante, permiten la aparición de crisis vitales de identidad.

\section{Referencias}

Arango, S. (2009). Una historiografía latinoamericana reciente sobre arquitectura y ciudad. Revista Diseño en síntesis 40-41(20), 32-43. https://www.academia.edu/30606712/ Una historiograf\%C3\%ADa latinoamericana reciente_sobre_arquitectura_y_ciudad

Arnoletto, E. J. (2007). Glosario de Conceptos Políticos Usuales. EUMEDNET. [enciclopedia virtual]. Definición de Líneas de Ruptura ("cleavage"). Argentina: Diccionarios en Internet de las Ciencias Económicas y Sociales. http://www.eumed.net/diccionariodefinicion. php?dic $=3 \&$ def $=376$

Barona, G. y Rojas, T. (2007) Falacias del pluralismo jurídico y cultural en Colombia. Popayán: Editorial Universidad del Cauca.

Bautista, N. (2011). Proceso de la investigación cualitativa; Epistemología, metodología y aplicaciones. Bogotá: Manual Moderno.

Bourdieu, P. (2002). La distinción: Criterios y bases sociales del gusto. (Traducción de María del Carmen Ruiz de Elvira). México: Taurus.

Bourdieu, P. (2007). El sentido práctico. Madrid: Ed. Siglo XXI.

Bourdieu, P. (2014). ¿Que significa hablar? Economia de los intercambios lingüisticos. Buenos Aires: Akal.

De Certeau, M., Giard, L., y Mayol, P. (1999). La invención de lo cotidiano: Habitar, cocinar. México: Universidad Iberoamericana.

Domínguez, M. y Portellano, C. (2011). La infancia y el estilo de vida en el espacio urbano madrileño. En Antonio Lucas Marín, María Dolores Cáceres y Enrique Morales Corral (ed.). Madrid ante los desafíos socia- les actuales, la realidad social de Madrid. Vol. III. Madrid:Fragua.

Duque, J. P. (2012). Territorios indígenas y Estado. Bogotá: Universidad Nacional de Colombia.

Duque, J. P., Salazar, O. y Castaño, G. (2004). Saminashi, arquitectura y cosmogonía en la construcción Kogi. Bogotá: Editorial Universidad Nacional de Colombia.

Fonseca, L. y Saldarriaga, A. (1992). Arquitectura popular en Colombia, herencias y tradi ciones. Bogotá: Altamir.

Foucault, M. (1999). El orden del discurso. Barcelona: Tusquets.

Gutiérrez-Aristizábal, A. (2017). La noción de paisaje social Un posible recurso para la valoración patrimonial. Revista de Arquitectura (Bogotá), 19(2), 16-27. https://doi.org/10.14718/RevArq.2017.19.2.855

Gutiérrez, G. (2012). Las aventuras de la memoria: proyecto de divulgación y capacitación para la salvaguardia del patrimonio arquitectónico. En Paisaje cultural urbano e identidad territorial $2^{\circ}$ Coloquio Internacional RIGPAC, Vol. II (p. 988-1000). Florencia: Aracne.

Gutiérrez, V. (1958) El país rural colombiano: ensayo de interpretación. Revista Colom biana de Antropología. Organo del instituto. VII. 1-126. Bogotá: Instituto Colombiano de Antropología, Ministerio de Educación Nacional.

http://biblioteca.icanh.gov.co/DOCS/MARC/ texto/REV-0915v7a01.pdf
Harris, M. (1982). Materialismo cultural. Madrid: Alianza.

Koselleck, R. (2012). Historias de conceptos. Estudios sobre semántica y pragmática del lenguaje político y social. Madrid: Trotta.

Lipset, S. M., y Rokkan, S. (1967). Cleavage structures, party systems and voter alignments: An introduction. En S. M. Lipset y S. Rokkan (eds.), Party systems and voter alignments: cross-national perspectives. New York: Free Press.

http://garfield.library.upenn.edu/ classics1990/A1990CR76700001.pdf

Nora, P. (2008). Los lugares de la memoria. Santiago de Chile: Trilce.

Rapoport, A. (1972). Vivienda y cultura. BarceIona: Gustavo Gilli.

Restrepo, E. (2012). Intervenciones en teoría cultural. Popayán: Universidad del Cauca.

Sánchez-Prieto, J. M. (2012). Reinhart KoseIleck: La Interdisciplinariedad de la historia. Memoria y Civilización 14, 475-499. https://www.unav.edu/publicaciones/ revistas/index.php/myc/article/view/1745

Silva, R. (2012). Republica liberal, intelectuales y cultura popular. Medellín: La Carreta.

Solo, M. (1987) Vivienda popular en un contexto arquitectónico. Segunda parte. Cuadernos de Escala, N. ${ }^{\circ}$ 14. Bogotá: Escala. 
Vol.

NrO. 2 REVISTA DE ARQUITECTURA

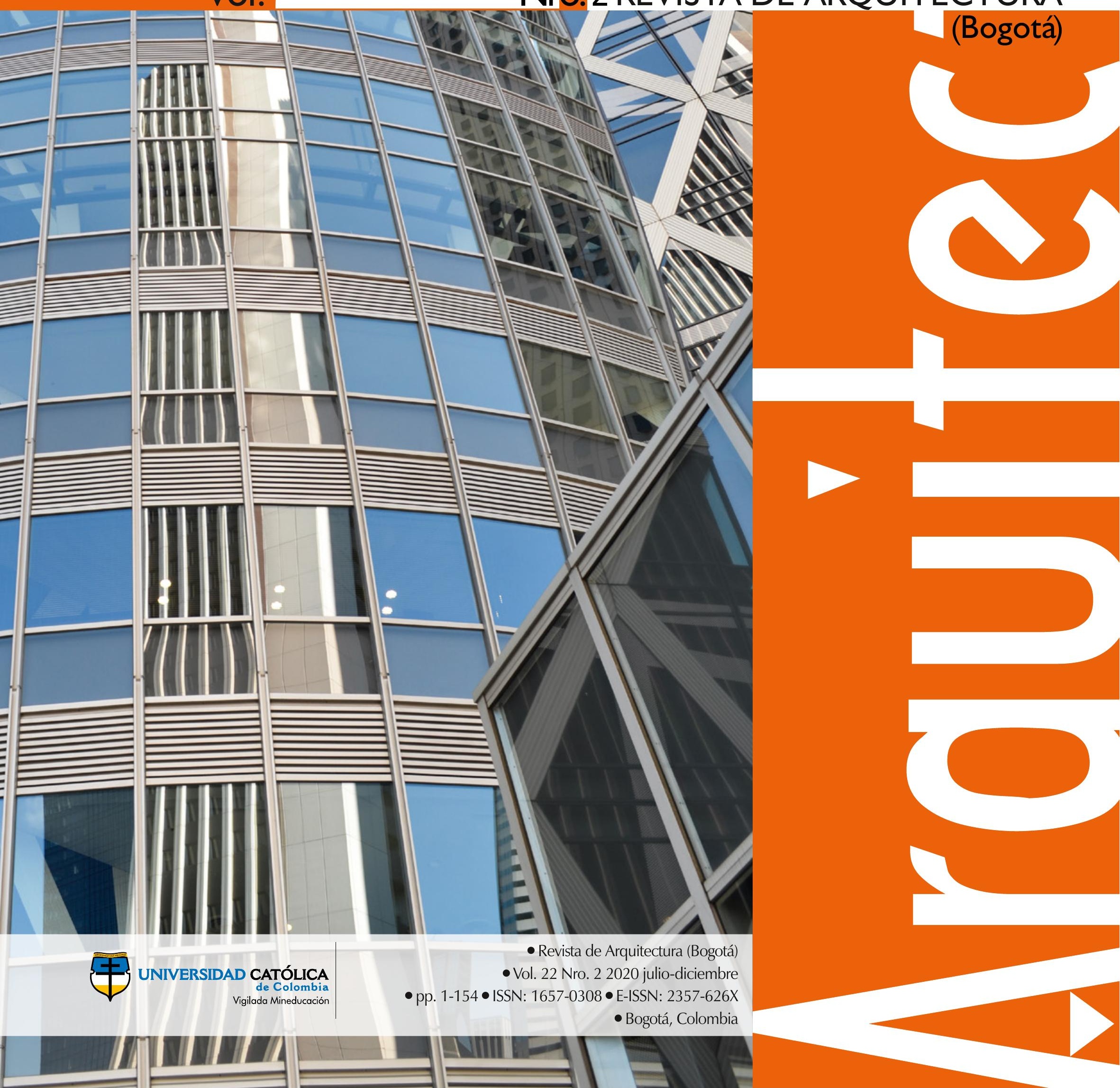


A Portada: Sokkuri (significa reflejo inverso) Mode Gakuen Cocoon Tower, Shinjuku, Tokyo, Japan Fotografía: Arquitecto José Tomás Pachajoa-Herrera (2012, diciembre)

\section{Enfoque y alcance}

La Revista de Arquitectura (Bogotá) ( (ISSN 1657-0308 Impresa y E-ISSN 2357-626X en línea) es una publicación científica seriada de acceso abierto, arbitrada mediante revisión por pares (doble ciego) e indexada, en donde se publican resultados de investigación originales e inéditos.

Está dirigida a la comunidad académica y profesional de las áreas afines a la disciplina. Es editada por la Facultad de Diseño y el Centro de Investigaciones (CIFAR) de la Universidad Católica de Colombia en Bogotá (Colombia).

La principal área científica a la que se adscribe la Revista de Arquitectura (Bogotá) según la OCDE es:

Gran área: 6. Humanidades

Área: 6.D. Arte

Disciplina: 6D07. Arquitectura y Urbanismo

También se publican artículos de las disciplinas como 2A02, Ingeniería arquitectónica; 5G03, Estudios urbanos (planificación y desarrollo); 6D07, Diseño.

Los objetivos de la Revista de Arquitectura (Bogotá) son:

- Promover la divulgación y difusión del conocimiento generado a nivel local, nacional e internacional

- Conformar un espacio para la construcción de comunidades académicas y la discusión en torno a las secciones definidas.

- Fomentar la diversidad institucional y geográfica de los autores que participan en la publicación.

- Potenciar la discusión de experiencias e intercambios científicos entre investigadores y profesionales.

- Contribuir a la visión integral de la arquitectura, por medio de la concurrencia y articulación de las secciones mediante la publicación de artículos de calidad.

- Publicar artículos originales e inéditos que han pasado por revisión de pares, para asegurar que se cumplen las normas éticas, de calidad, validez científica, editorial e investigativa.

- Fomentar la divulgación de las investigaciones y actividades desarrolladas en la Universidad Católica de Colombia
Palabras clave de la Revista de Arquitectura (Bogotá): arquitectura, diseño, educación arquitectónica, proyecto y construcción, urbanismo.

Idiomas de publicación: español, inglés, portugués y francés.

Título abreviado: Rev. Arquit.

Titulo corto: RevArq

\section{Políticas de sección}

La revista se estructura en tres secciones correspondientes a las líneas de investigación activas y aprobadas por la institución, y dos complementarias, que presentan dinámicas propias de la Facultad de Diseño y las publicaciones relacionadas con la disciplina.

Cultura y espacio urbano. En esta sección se publican los artículos que se refieren a fenómenos sociales en relación con el espacio urbano, atendiendo aspectos de la historia, el patrimonio cultural y físico, y la estructura formal de las ciudades y el territorio.

Proyecto arquitectónico y urbano. En esta sección se presentan artículos sobre el concepto de proyecto, entendido como elemento que define y orienta las condiciones proyectuales que devienen en los hechos arquitectónicos o urbanos, y la forma como estos se convierten en un proceso de investigación y nuevo de conocimiento. También se presentan proyectos que sean resultados de investigación, los cuales se validan por medio de la ejecución y transformación en obra construida del proceso investigativo. También se contempla la publicación de investigaciones relacionadas con la pedagogía y didáctica de la arquitectura, el urbanismo y el diseño.

Tecnología, medioambiente y sostenibilidad. En esta sección se presentan artículos acerca de sistemas estructurales, materiales y procesos constructivos, medioambiente y gestión, relacionados con los entornos social-cultural, ecológico y económico.

Desde la Facultad. En esta sección se publican artículos generados en la Facultad de Diseño, relacionados con las actividades de docencia, extensión, formación en investigación o internacionalización, las cuales son reflejo de la dinámica y de las actividades realizadas por docentes, estudiantes y egresados; esta sección no puede superar el $20 \%$ del contenido.

Textos. En esta sección se publican reseñas, traducciones y memorias de eventos relacionados con las publicaciones en Arquitectura y Urbanismo.
A Frecuencia de publicación

Desde 1999 y hasta el 2015, la Revista de Arquitectura (Bogotá) publicó un volumen al año, a partir del 2016 se publicarán dos números por año en periodo anticipado, enero-junio y julio-diciembre, pero también maneja la publicación anticipada en línea de los artículos aceptados (versión Post-print del autor).

La Revista de Arquitectura (Bogotá) se divulga mediante versiones digitales (PDF, HTML, EPUB, XML) e impresascon un tiraje de 700 ejemplares, los tiempos de producción de estas versiones dependerán de los cronogramas establecidos por la editorial.

Los tiempos de recepción-revisión-aceptación pueden tardar entre seis y doce meses dependiendo del flujo editorial de cada sección y del proceso de revisión y edición adelantado.

Con el usuario y contraseña asignados, los autores pueden ingresar a la plataforma de gestión editorial y verificar el estado de revisión, edición o publicación del artículo.
A Canje

La Revista de Arquitectura (Bogotá) está interesada en establecer canje con publicaciones académicas, profesionales o científicas del área de Arquitectura y Urbanismo, como medio de reconocimiento y discusión de la producción científica en el campo de acción de la publicación.

\section{Mecanismo}

Para establecer canje por favor descargar, diligenciar y enviar el formato: RevArq FP20 Canjes

Universidad Católica de Colombia (2020,
julio-diciembre). Revista de
Arquitectura (Bogotá), 22(2),
I-I52. Doi: 10.14718

ISSN: 1657-0308
E-ISSN: 2357-626X
Especificaciones:
Formato: 34 x $24 \mathrm{~cm}$
Papel: Mate II5 g
Tintas: Negro y policromía

Contacto

Dirección postal:

Avenida Caracas No. 46-72.

Universidad Católica de Colombia

Bogotá D.C.(Colombia)

Código postal: 111311

Facultad de Diseño

Centro de Investigaciones (CIFAR).

Sede El Claustro. Bloque "L", 4 piso

Diag. 46A No. $15 b-10$

Editor, Arq. César Eligio-Triana

Teléfonos:

+57 (1) $3277300-3277333$

Ext $3109 \cdot 311205146$

Fax: + 57 (1) 2858895
Correo electrónico:

revistadearquitectura@ucatolica.edu.co cifar@ucatolica.edu.co

Página WEB:

www.ucatolica.edu.co vínculo Revistas científicas

http://publicaciones.ucatolica.edu.co revistas-cientificas http://editorial.ucatolica.edu.co/ojsucatolica/revistas_ucatolica/index.php/RevArq 


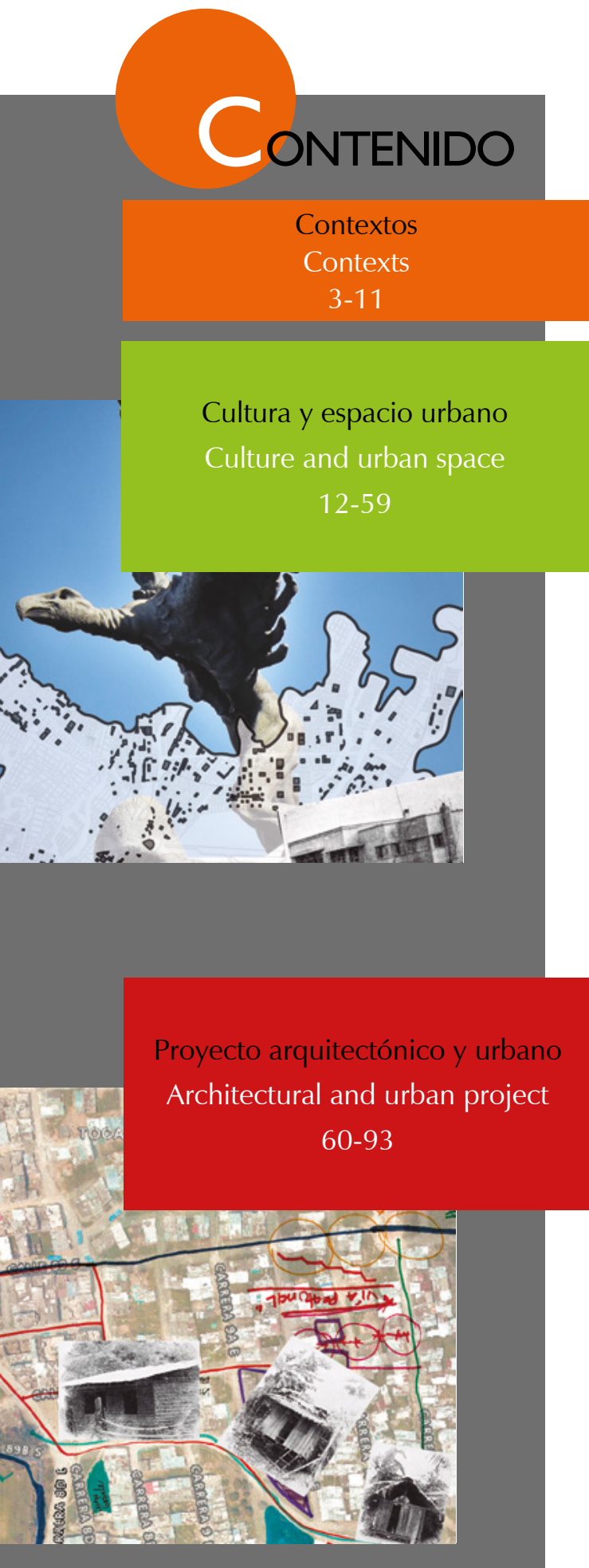

Tecnología, medioambiente y sostenibilidad

Technology, environment and sustainability 94-152

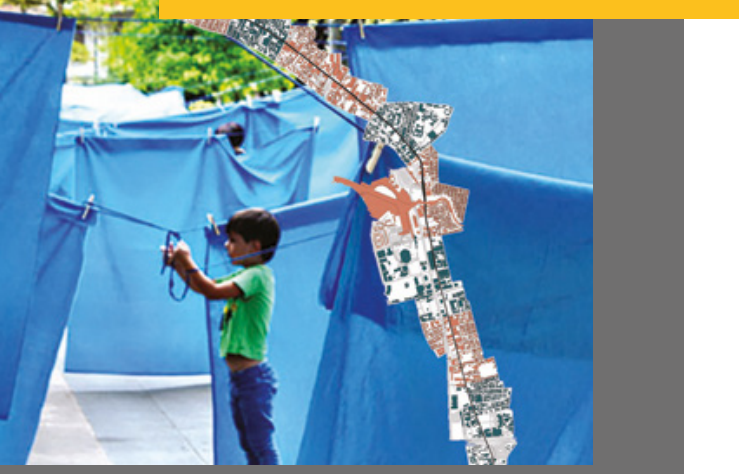

Espacio y tiempo del taller de proyectos arquitectónicos:

la Escuela de Arquitectura de Valencia

Débora Domingo-Calabuig

Laura Lizondo-Sevilla

La comunidad local en la valoración del patrimonio rural.

La escuela Francisco Arias en Lavalle

Isabel Durá-Gúrpide

Matías J. Esteves

ES Hábitat Accesible. Desarrollo de modelos conceptuales urbano-habitacionales.

Alina Delgado-Bohórquez

ES Monumentos y arte urbano: percepciones, actitudes y valores en la ciudad de Manizales.

Rodrigo Santofimio-Ortiz

Sandra Milena Pérez-Agudelo

ES Nueva centralidad en interfase urbano-rural (I-UR)

Caso: sector Umapalca, zona sur de Arequipa Metropolitana

David Jesús Lovon-Caso

Ana de los Ángeles Larota-Sanz

ES Arquitecturas tradicionales y populares: un reto para la historiografía de la arquitectura en Colombia

Guillermo Gutiérrez-Morales

Mito o realidad.

Gustave Eiffel y el templo San Marcos de Arica

Darci Gutiérrez-Pinto

ES La caminabilidad como estrategia proyectual para

las redes peatonales del borde urbano.

Barrio Sierra Morena, Usme.

Marielena Medina-Ruiz

Siudades costeras e indicadores de sostenibilidad:

una aproximación desde el metabolismo urbano de la calle

El caso de la avenida Juan Ponce de León, en San Juan, Puerto Rico

María Helena Luengo-Duque

Fabricación de ladrillos con polvo-residuo de mármol en México. 106

Propiedades físicas y mecánicas del polvo-residuo de mármol

de la provincia de la Comarca Lagunera, en México

C. Ponce-Palafox
Julián Carrillo
A. López-Montelongo

Determinación de emisiones de Gases de Efecto Invernadero (GEI) en una matriz energética sustentable mediante análisis de escenarios.

Estudio de caso en zonas áridas con alto riesgo hídrico

Alba Inés Ramos Sanz

ES La participación infantil en proyectos urbanos.

El juego en espacios públicos para la promoción del aprendizaje

de conceptos ambientales

Carolina Polo-Garzón

Adriana Patricia López-Valencia

Alba Inés Ramos Sanz

ES Gestión territorial y sus implicaciones con el ODS11

Reflexiones desde Colombia y Costa Rica

Tania Giraldo-Ospina

Félix Zumbado-Morales 
Espacio y tiempo del taller de proyectos arquitectónicos:

la Escuela de Arquitectura de Valencia

ن Space and time of the design studio: the Architectu

La comunidad local en la valoración del patrimonio rural. La  escuela Francisco Arias en Lavalle

ن The local community in the appreciation of rural heritage. Francisco Arias ¿ school in Lavalle

Hábitat accesible. Desarrollo de modelos conceptuales

ij Affordable Habitat - The Development of Housing and Neighbourhood

Simulation Models

Monumentos y arte urbano: percepciones, actitudes y valores en la ciudad de Manizales

m ن Manizales $\stackrel{\infty}{+}$ Umapalca, zona sur de Arequipa Metropolitana

New Centrality in Rural Urban Interface. Case: Sector Umapalca, South Zone of Metropolitan Arequip

0

Arquitecturas tradicionales y populares: un reto para la

historiografía de la arquitectura en Colombia

ن Traditional and popular architectures: a challenge for architecture

这

¿ேं

\section{Ciudades costeras e indicadores de sostenibilidad:}

よ

caso de la avenida Juan Ponce de León, en San Juan, Puerto Rico

Coastal cities and sustainability indicators: an approach from the urban

metabolism of the street. The case of Juan Ponce de León avenue, in San Juan, Puerto Rico

Fabricación de ladrillos con polvo-residuo de mármol en México. - Propiedades físicas y mecánicas del polvo-residuo de mármol de 으 la provincia de la Comarca Lagunera, en México

ن Physical and mechanical properties of bricks with dust residue from marble in

México.Physical and mechanical properties of the marble dust-residue from the Comarca Lagunera Province, in Mexico

\section{Julián Carrillo}

Determinación de emisiones de Gases de Efecto Invernadero * (GEI) en una matriz energética sustentable mediante análisis de

- escenarios. Estudio de caso en zonas áridas con alto riesgo hídrico ن Determination of greenhouse gas emissions (GHG) in a sustainable energy matrix $\varangle \quad$ through scenario analysis. Case Study in arid zones with high hydric risk

\section{La participación infantil en proyectos urbanos. El juego en}

ㄴ espacios púb

$\stackrel{2}{\sim}$ ambientales

ن Children's participation in urban projects. Play in public spaces for the

$\varangle$ promotion of the learning of environmental concepts Gestión territorial y sus implicac
desde Colombia y Costa Rica

₹ desde Colombia y Costa Rica

Land Management and its implications with SDG II Reflections from

ن Colombia and Costa Rica

¿ Tania Giraldo-Ospina
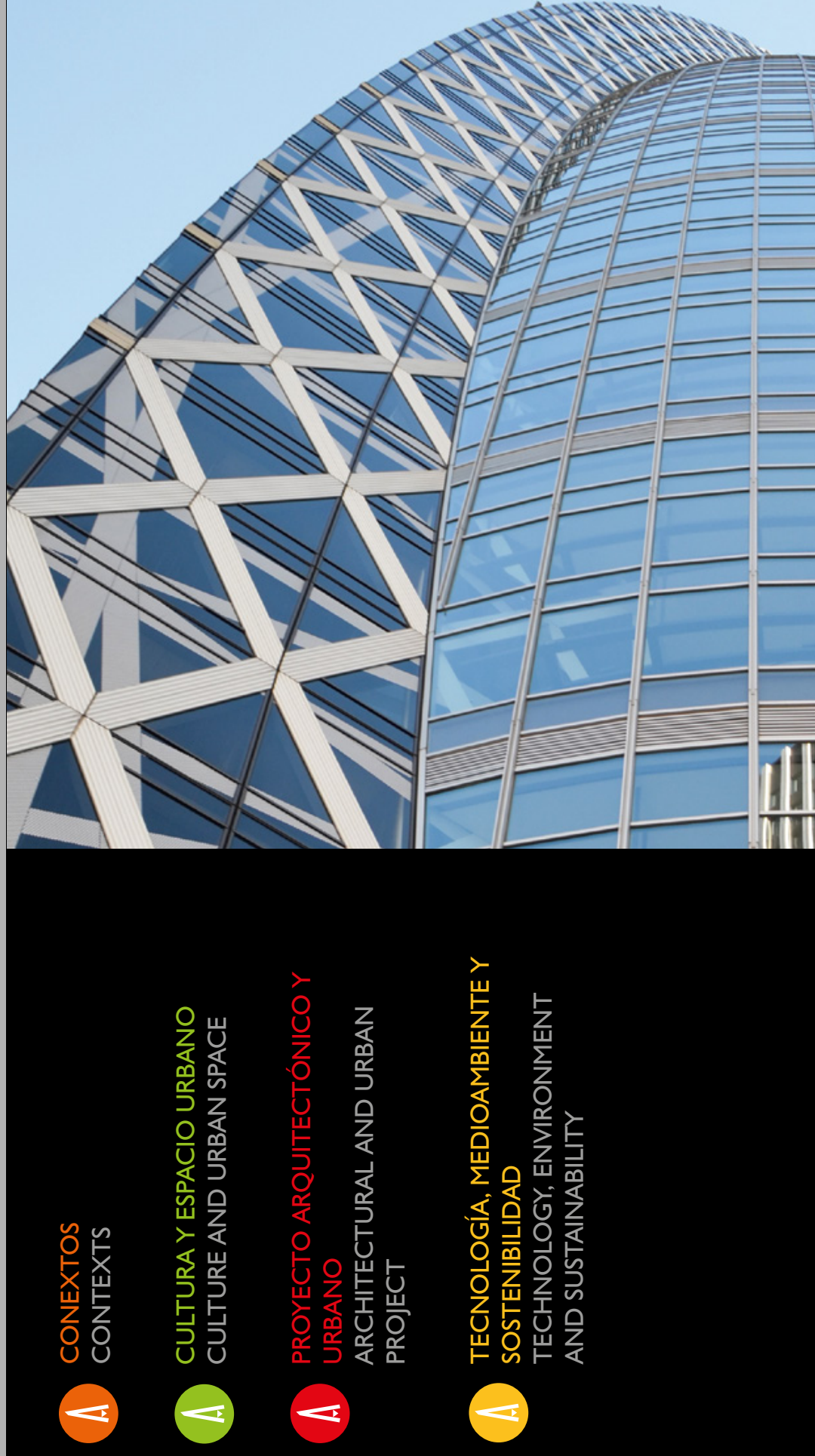

La Revista de Arquitectura es de acceso abierto, arbitrada e indexada y está presente en:

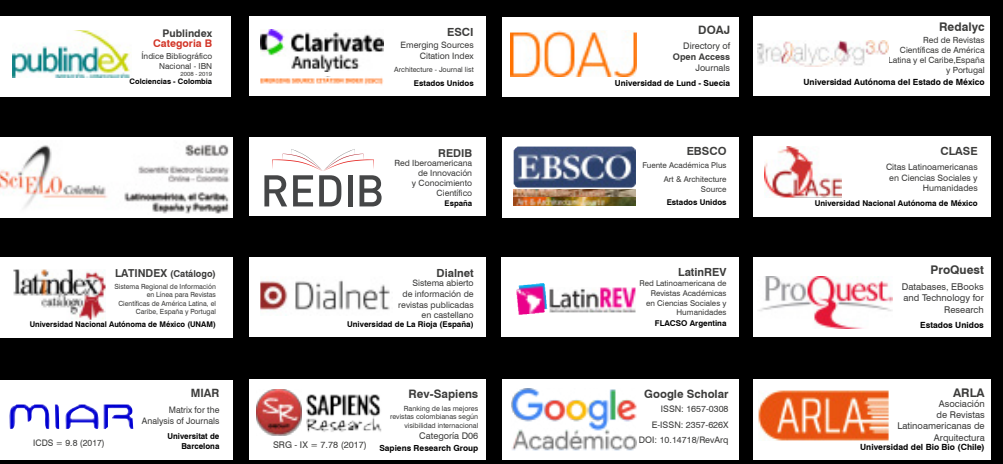

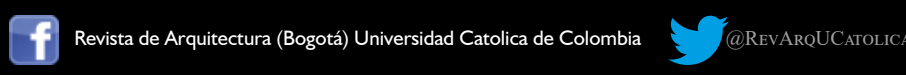

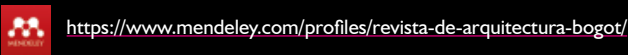

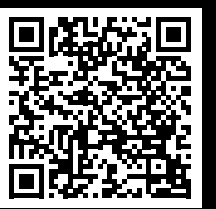

† UNIVERSIDAD CATÓLICA

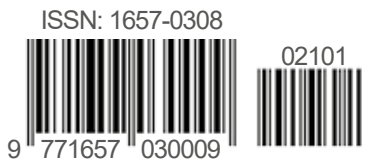

